\title{
Directed 2-dimensional organisation of collagen: Role of cross-linking and denaturing agents
}

\author{
NISHTAR NISHAD FATHIMA ${ }^{1}$, ARUNA DHATHATHREYAN $^{1, *}$ and \\ THIRUMALACHARI RAMASAMI ${ }^{2}$ \\ ${ }^{1}$ Chemical Laboratory, Central Leather Research Institute, Council of Scientific and Industrial Research, \\ Adyar, Chennai 600020 \\ ${ }^{2}$ Department of Science and Technology, New Mehrauli Road, New Delhi 110016 \\ e-mail: aruna@clri.res.in
}

MS received 19 January 2010; revised 19 May 2010; accepted 12 June 2010

\begin{abstract}
The effect of additives like curcumin and surfactants on the self-assembly of collagen from a simple 2-dimensional system of Langmuir films of the protein at air/solution interface has been attempted in this study using quartz crystal microbalance (QCM) and dynamic surface tensiometer. Though pure curcumin is not surface active, a synergistic effect of collagen with curcumin seems to lead to enhanced surface activity in the protein. In general, the presence of additives, increases the surface activity of collagen even for the lowest concentration and the largest change in surface activity is seen for collagen with sodium dodecyl sulfate (SDS). The results suggest interplay between the unexposed hydrophobic groups, and the opening out and solvation of the more charged or polar groups at the surface leading to aggregation followed by self-assembly. Modulation of aggregation at interface in collagen due to these additives may be an approach that could be explored for possible applications in bio-materials and for delivery of protein-drug complexes.
\end{abstract}

Keywords. Collagen; surface tension; fibril formation; quartz crystal microbalance (QCM); selfassembly; curcumin; surfactants.

\section{Introduction}

Collagen is the major structural protein of the extracellular matrix. The current medical applications of collagen as biodegradable material are associated with its natural properties including low immune response, the ability to promote cellular growth, attachment homeostasis and the ability of collagen solution to reconstitute in vitro into the microfibrillar structure normally found in natural tissues. ${ }^{1}$ Solubilised collagen self-assembles at $37^{\circ} \mathrm{C}$ to form rigid gels. ${ }^{2,3}$ Under these conditions collagen molecule aggregates spontaneously into fibrillar assemblies with a characteristic $67 \mathrm{~nm}$ fibril repeat distance as observed via electron microscopy and $\mathrm{X}$-ray diffraction. ${ }^{4}$ A number of papers on the effects of solvent conditions such as concentration, temperature and ionic strength on the final morphology of the products and an exhaustive description of the process have appeared in the literature. ${ }^{5,6}$ Studies on

\footnotetext{
*For correspondence
}

the self-assembly and dynamic evolution of collagen gels from semidilute solutions in a microfluidic device have been reported recently by Köster $e t a l{ }^{7}$

A number of unanswered questions concerning folding and other changes in secondary structures of proteins forming stable aggregate structures still exist. ${ }^{8}$ The exact contributions from electrostatic, dispersive and $\mathrm{H}$-bonding forces are also not completely understood. An improved understanding and characterization of the assembly and organization of fibrillar structures in collagen may therefore be feasible using a simple 2-dimensional system of Langmuir films of the protein at air/solution interface. It is well-established that in vitro aggregation of proteins is often influenced by a number of experimental factors, including primary structure, secondary structure, temperature, $\mathrm{pH}$, and protein concentration.

Hence, in this study an attempt has been made to understand the 2D self organizational process of collagen using Langmuir films at air/solution interface and relate to the morphology of the assembled product using confocal microscope. Further, an effect of 
additives namely, curcumin and surfactants on the surface property of collagen has been studied. Curcumin has attracted considerable attention in recent years due to its wide spectrum of biological and pharmacological activities, including antioxidant, antitumor, anti-inflammatory, antibacterial, antifungal, antiviral, antiproliferative, anti-invasive, antiangiogenic and anticoagulant activities. ${ }^{9-12}$ The interaction between surfactants and proteins has important consequences for many processes in the chemical, biochemical, petrochemical, and pharmaceutical industries, especially in solubility, rheological properties, and colloid stability of systems.

\section{Experimental}

\subsection{Materials and collagen solutions preparation}

Deionized distilled water from Millipore (Milli-Q) $(18 \mathrm{M} \Omega$ ) was used for the experiments. All the fluids used in the study were HPLC grade and obtained from Merck, India. Curcumin (figure 1) was obtained from Sigma Chemicals, USA. Collagen solutions for dynamic surface tension measurements were prepared from tendons freshly dissected from the tails of 6-month-old male albino rats frozen at $-20^{\circ} \mathrm{C}$ by acetic acid extraction and salting-out with $\mathrm{NaCl}^{13}$ The purity of the collagen preparation was confirmed by sodium dodecyl sulfate-polyacrylamide gel electrophoresis; the bands appearing in the gel corresponded only to type 1 collagen. The collagen concentration in the solutions was determined from the hydroxyproline content. ${ }^{14}$ The average molecular weight of collagen was 300,000 Daltons, on the basis of which the molar concentration was determined. The stock concentration of the prepared collagen was $0 \cdot 2 \mu \mathrm{M}$. Collagen solution was treated with curcumin in the increasing molar ratio of collagen to curcumin from $1: 1$ to $1: 100$ and incubated for $24 \mathrm{~h}$ under room temperature

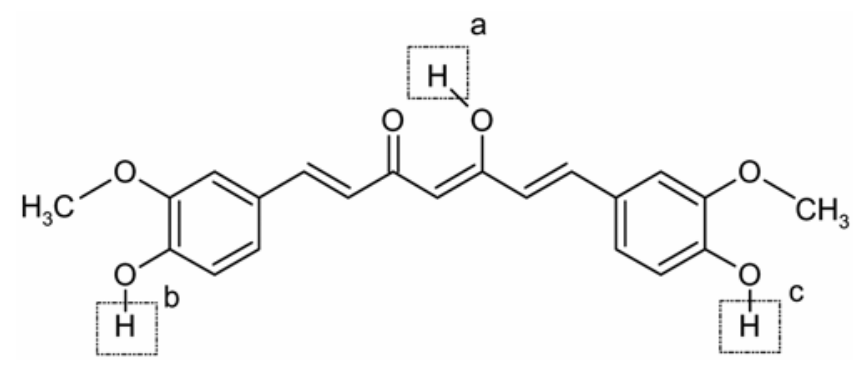

Figure 1. Structure of curcumin. $\left(25^{\circ} \mathrm{C}\right)$. Collagen solution was also treated with three surfactants viz. SDS, CTAB and Triton X-100 in increasing molar ratio of collagen to surfactant as $1: 1,1: 10,1: 100$ and incubated for $24 \mathrm{~h}$ under room temperature $\left(25^{\circ} \mathrm{C}\right)$.

Collagen solution for Langmuir-Blodgett studies was prepared from ray fish skin as described below. Fish collagen is selected because of its low melting temperature. Fish collagen has less number of hydroxyproline when compared to that of the other species. Hydroxyproline is responsible for the intermolecular hydrogen bonding, which results in the fibril formation. Since the rate of formation of fibril is expected to be lowered, experimental measurements using the conventional techniques is relatively easier. The melting temperature of fish collagen is around $20-22^{\circ} \mathrm{C}$. This affords a feasibility to carry out measurements under controlled temperature conditions.

The cleaned fish skin is soaked in chloroform: methanol mixture in the ratio of $1: 3$ and kept at $4{ }^{\circ} \mathrm{C}$ overnight. The fish skin is taken out and the flesh is removed to some extent and then soaked in $0.5 \mathrm{M}$ sodium acetate and kept at $4^{\circ} \mathrm{C}$ overnight. Next day the flesh is completely removed and is soaked in $0.5 \mathrm{M} \mathrm{NaCl}$ for two days. The $\mathrm{NaCl}$ solution is changed for every $6 \mathrm{~h}$. At the end of the second day the skin becomes very soft, it is then cut into small pieces. The cut pieces are then washed a number of times with cold distilled water this is to ensure that $\mathrm{NaCl}$ is completely removed. The washed pieces are then soaked in $0.5 \mathrm{M}$ Acetic acid and the set-up is kept for stirring for the next two days in the cold room. The collagen solution is now prepared by acetic acid extraction and salting-out with $\mathrm{NaCl}{ }^{13}$ The concentration of the collagen was $1.6 \mathrm{mg} / \mathrm{ml}$ with molar mass of 320,000 Daltons.

\subsection{Surface tension measurements}

The surface tension measurements were carried out with a single barrier NIMA trough (model no. 610S) attached with a thermostat. The experiments were carried out at $T=22^{\circ} \mathrm{C}$. For every concentration of collagen used, the solution prepared from the stock (concentration $10^{-4} \mathrm{M}$ ) was filled in the trough. The surface was cleaned using an aspirator at the beginning of each experiment. The surface pressure at the beginning of each experiment was checked before letting the collagen molecules organize at the air/solution interface. The change in the surface 
pressure value as a function of time was carried out for all the concentrations of collagen and 2 different $\mathrm{pH}$ values 3.8 and 7.6. The results reported here are the averages of at least 5 measurements. Two sets of experiments were carried out one in which the concentration of collagen in the sub-phase was varied from $10^{-9} \mathrm{M}$ to a maximum concentration of $10^{-7} \mathrm{M}$ at $\mathrm{pH}=3.8$. In another experiment for the lowest concentration of collagen, the $\mathrm{pH}$ of the solution was varied from 3.0 to a maximum of 7.8 . The buffer solutions used were acetic acid buffer (for $\mathrm{pH}$ between 3 and 4.5 and phosphate buffer for $\mathrm{pH}$ between 5 and 7.8 (concentrations of buffer around $1 \mathrm{mM})$.

A NIMA DST 9005 contact angle meter was used to measure the dynamic surface tension of the fluids (accuracy $\pm 0.01 \mathrm{mN} / \mathrm{m}$ ) (by using a Du-Nuoy ring) on glass. DST9005 Dynamic Surface Tensiometer is a sophisticated computer controlled instrument that can measure the forces on samples as they are immersed in the test liquid. Advancing and receding, dynamic and static contact angles can be measured. A temperature controllable stage and stirrer agitator are included for precise, repeatable measurements.

Glass substrates were cover slips $(18 \mathrm{~mm} \times$ $18 \mathrm{~mm}$ ) with a mean $\mathrm{rms}$ value of about $0.16 \pm$ $0.01 \mathrm{~nm}$ from ERMA, FRG and were cleaned with warm chromic acid and washed repeatedly with fresh deionized water, dried using a stream of nitrogen and were stored in a desiccator for use. The cover slips were immersed in a Perspex tank where the different sample liquids or their mixtures were kept. Both dynamic and static surface tension measurements were carried out and the results were recorded on a computer.

\subsection{Confocal measurements}

Sodium salt of ANS was used at a concentration of $10^{-7} \mathrm{M}$ to stain the collagen. Assemblies of collagen from dilute solution at the interface have been transferred to solid substrates and Leica SP2 model Confocal microscope in emission mode has been used to analyse the morphology.

\subsection{Quartz crystal microbalance (QCM) measurements}

The measurements were performed with a RQCM instrument from Maxtek, USA. The QCM sensors with gold electrodes were polished crystals with a fundamental frequency of about $5 \mathrm{MHz}$. The gold coated quartz crystals were cleaned in piranha solution for a few minutes, rinsed with water and then stored in a dessicator. The crystal $(22 \mathrm{~mm}$ in diameter) was coated using ethanolic solution of with decane thiol. The QCM experiments were performed in static conditions at $25^{\circ} \mathrm{C}$. Before adsorption, the cell was filled with buffer to record a baseline. The response of QCM sensor was monitored as a function of time after adding collagen solution. The adsorption amount of collagen, $\Delta m$, can detect with frequency decrease of QCM, $\Delta F$ by Sauerbrey's equation ${ }^{15}$ as follows

$$
-\Delta F=\frac{2 F_{0}^{2}}{A \sqrt{\rho_{q} \mu_{q}}} \times \Delta m,
$$

where $F_{0}$ is the fundamental frequency of QCM $(5 \mathrm{MHz}), \mathrm{A}$ is the electrode area $\left(1.6 \mathrm{~cm}^{2}\right), \rho_{\mathrm{q}}$ is the density of the quartz and $\mu_{\mathrm{q}}$ is the shear modulus.

\subsection{Turbidity measurements}

Turbidity curves monitoring the process of selfassembly of collagen were obtained using the optical density at $313 \mathrm{~nm}$ as a function of time on a Perkin Elmer Lambda spectrophotometer attached with a Peltier temperature controller. The sample solutions were prepared by the addition of adequate volume of acetic acid $(5 \mathrm{mM})$ soluble collagen, buffer and curcumin. The final volume of the sample was made up to $1200 \mu \mathrm{L}$ after the addition of required amount of $0 \cdot 1 \mathrm{~N} \mathrm{NaOH}$ to obtain a $\mathrm{pH}$ of $7 \cdot 2-7 \cdot 4$. The temperature of the cells was maintained at $35^{\circ} \mathrm{C}$. Turbidity measurements of samples were recorded immediately after the addition of $\mathrm{NaOH}$ for $10 \mathrm{~min}$. The final concentration of collagen was $1.25 \mu \mathrm{M}$. Final concentration of buffer was maintained at $10 \mathrm{mM}$ $\mathrm{NaH}_{2} \mathrm{PO}_{4}, 10 \mathrm{mM} \mathrm{Na} \mathrm{HPO}_{4}, 150 \mathrm{mM} \mathrm{NaCl}$. The plots were fitted to sigmoidal function after normalizing it to zero.

\section{Results and discussion}

\subsection{Studies on self-assembly of collagen using surface tension as a parameter}

2D Gibb's systems make an attractive model to study organized assemblies of proteins because of the natural constraints that amphiphilic systems 
exhibit in terms of definite orientation at the interface (hence definite thickness) and controllable surface concentrations in the $X-Y$ plane. Very dilute solutions of proteins when allowed to assemble at the interface should initiate the fibril formation. These 2-D assemblies have been studied using a Langmuir Film balance. In cold and acidic solutions, collagen exhibits solubility and is found mainly in the monomeric form. However, a weak surface activity for pure collagen is expected. Therefore, here surface tension is used as a parameter to probe the assembly processes of collagen at solution/air interface.

For different concentrations of collagen in solution ranging from $5.6 \times 10^{-9}$ to $2.3 \times 10^{-7} \mathrm{M}$, surface tension as a function of time was measured. Figures $2 \mathrm{a}$ and $\mathrm{b}$ show plots for the lowest and highest
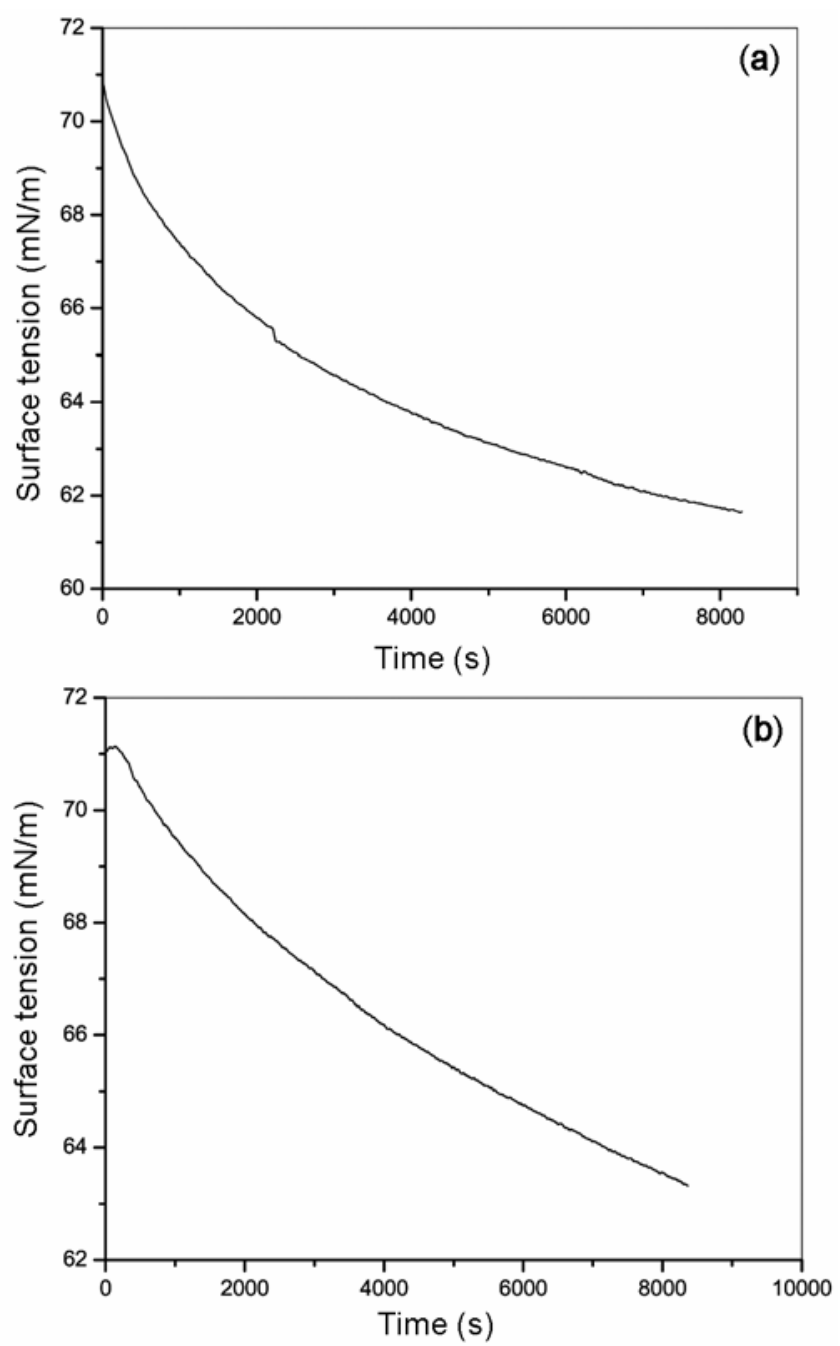

Figure 2. (a) Surface tension $(\gamma)$-time plot for lowest concentration $\left(5.6 \times 10^{-9} \mathrm{M}\right)$, (b) surface tension $(\gamma)$-time plot for highest concentration $\left(2 \cdot 3 \times 10^{-7} \mathrm{M}\right)$ for fish collagen $\left(T=22^{\circ} \mathrm{C}\right)$. concentrations of collagen at a sub-phase $\mathrm{pH}$ of $3 \cdot 8$. A rate process and a corresponding model for the process was obtained by plotting instantaneous rate (evaluated by plotting $\log (\delta \gamma / \mathrm{d} t)$ vs $\log$ time) vs instantaneous time for range of concentrations. The rate plots (figure 3 ) suggest that at $\mathrm{pH} 3.8$ the fibril formation follows first order kinetics. This also suggests that in organization of protein, the extent of solvation and synergy between these two are necessary first steps for fibril formation.

In order to check the formation of fibrils, Gibb's film of the protein has been analysed at the interface using confocal microscopy (figure 4). We observed protein structures with different sizes, revealing a high degree of conformational heterogeneity of the fibrils upon self-assembling in the buffer medium. Figure $4 \mathrm{a}$ shows formation of spherical aggregated structures and in figure $4 \mathrm{~b}$, the banded pattern of the aggregated fibrils are seen. Spatially resolved fluorescence experiments revealed the typical structural features of the fibril. Figure $4 \mathrm{a}$ shows a region of high fibril density (near the edge of the deposited drop), in which the presence of large amorphous aggregates seem to act as fibrillogenesis nuclei, from which most of the fibrillar structures radiate. The diameter of the fibrils was found to be in the $250 \mathrm{~nm}$ range. The presence of some monomers or small aggregates also is visible in the background of the image (figure $4 \mathrm{~b}$ ). These protein aggregates are thought to fuse into fibrillar structures, although the detailed mechanism underlying the fibrillogenesis process is not yet completely understood. ' $D$ ' spacing of the bands seen in this structure lie between 52

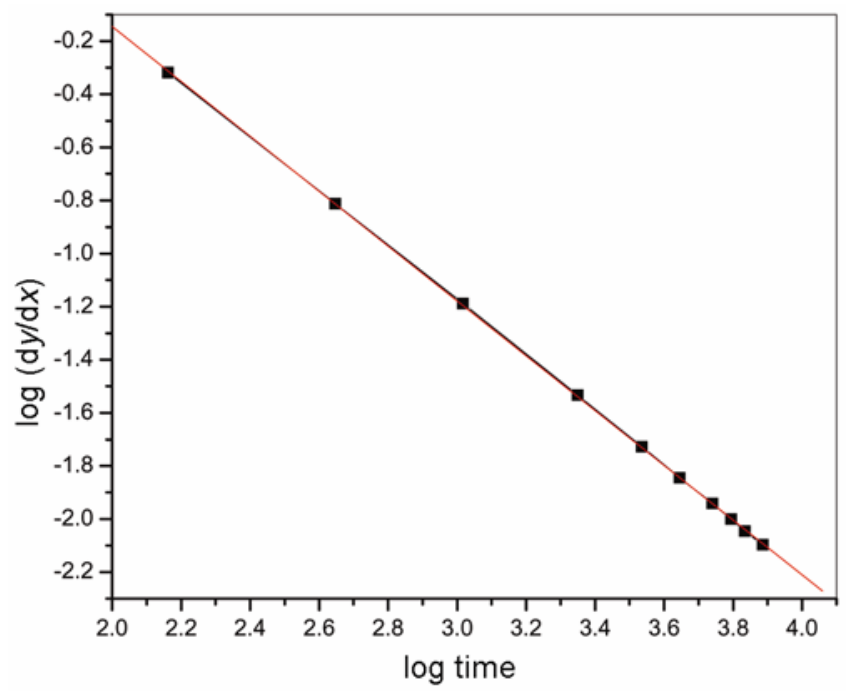

Figure 3. Rate plot evaluated by plotting $\log (\delta \gamma / \mathrm{d} t)$ vs log time ( -experimental data, — fit) for fish collagen. 

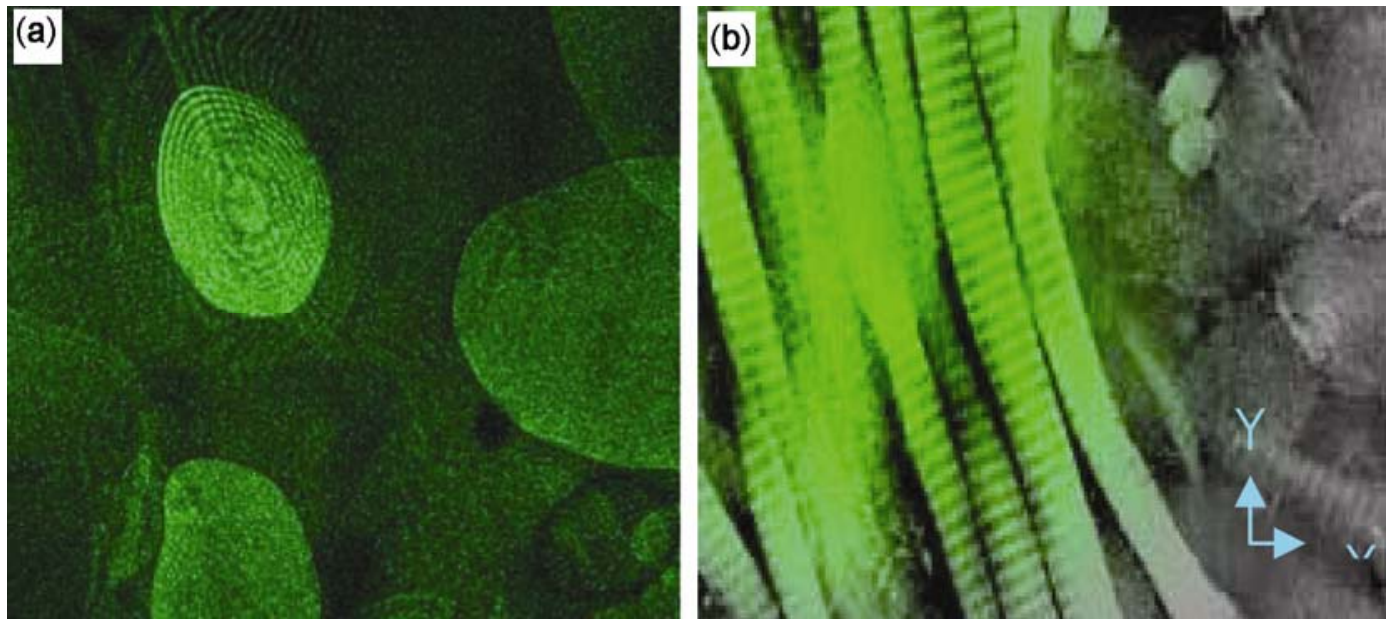

Figure 4. Two representative confocal images of fish collagen stained with ANS. (a) Confocal micrograph of fibrils from lowest concentration of collagen $(X \times Y$ scan 1.5 $\mu$ ), (b) Aggregated structures of fibrils $(X \times Y \operatorname{scan} 1.0 \mu)$.

and $59 \mathrm{~nm}$. Earlier reported values of the periodicity on dry fibril is $67 \mathrm{~nm}$ and a lower value in water between 54 and $57 \mathrm{~nm}$ could be due to complex space transformations of tropocollagen orientation caused by water saturation and swelling. Due to the experimental set-up of confocal microscopy where the sample substrate is held using film of oil at the objective, local changes in polarity as well as drying could lead to the changes in the ' $\mathrm{D}$ ' spacing values seen here.

\subsection{Surface tension changes in collagen on interaction with curcumin and surfactants}

In order to study the effect of a crosslinking agent like curcumin on the organization of the protein at the interface a study was undertaken with rat tail tendon (RTT) collagen with curcumin. Figure 5a shows the surface tension plots of collagen treated with curcumin, as a function of time for the lowest and highest concentrations. It is seen that the rate of change in surface tension for a collagen to curcumin ratio of $1: 1$ and $1: 100$ remains almost the same. However, the initial values of surface tension reported here show clearly that on increasing concentration of curcumin the surface tension of collagen decreases rapidly $(48 \mathrm{mN} / \mathrm{m})$ indicating that the protein now becomes more surface active. This is due to curcumin forcing more non-polar groups to get exposed and thereby forcing the protein to arrive at the interface and thereby altering the surface tension. Figures $5 \mathrm{~b}-\mathrm{d}$ show the surface tension plots of collagen as a function of time with additives CTAB,
SDS and Triton X-100, respectively for lower and higher concentrations. All the surfactants cause an increase in surface activity of the protein with maximum change occurring in the presence of anionic surfactant, SDS. Here, mixed collagen films with different surfactants like SDS, CTAB and Triton X-100 have been used. Surfactants are known to act as denaturants. ${ }^{16}$ Since both crosslinking agent like curcumin and denaturants like surfactants are decreasing the surface tension of the protein, there may be two different mechanisms operating to influence the surface activity. Curcumin is likely to alter the hydration network around the protein while denaturants break the water structure. This alteration of the water network could lead to more stability of the hydrated protein and this has been seen in the increase in shrinkage temperature of collagen after crosslinking with curcumin. ${ }^{17}$

The synergistic effect of the additive and collagen to the surface properties are best described by rate of change of surface tension. All the surfactants show a slow rate for lower concentration and then an increase in the rate. This suggests that at lower concentration, the surfactants adsorb to available sites without possibly altering any local hydrated structures drastically. In case of SDS the rate seems to be low. This could be due to the fact that anionic (SDS) surfactant facilitates the micelle formation of collagen by enhancing the hydrophobic interaction while CTAB may hinder the micelle formation of the collagen owing to the repulsive effect between $\mathrm{N}^{+}$ion of CTAB and $\mathrm{NH}_{4}^{+}$ion of amino acids. The addition of varying amounts of curcumin to suspensions of 

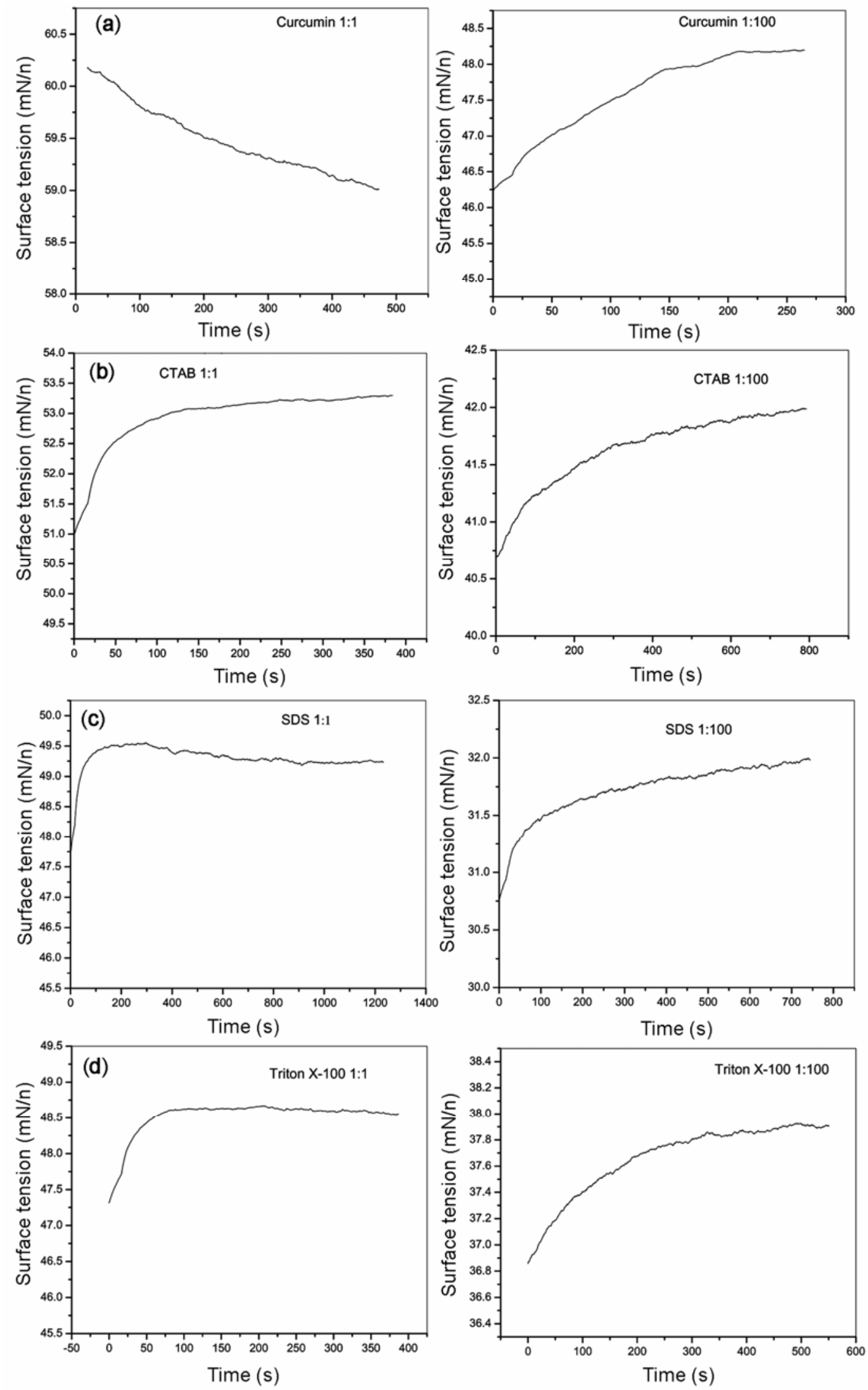

Figure 5. Dynamic surface tension plots for (a) curcumin, (b) CTAB, (c) SDS, (d) TRITON X-100 treated RTT collagen (for lowest and highest concentrations of the additives, $1: 1$ and $1: 100$ of collagen : additive). 
Table 1. Static surface tension values of native collagen and collagen treated with various concentrations of additives.

\begin{tabular}{llcccc}
\hline & & \multicolumn{3}{c}{ Static surface tension $(\mathrm{mN} / \mathrm{m})$} \\
\cline { 3 - 5 } S1. no. & \multicolumn{1}{c}{ Sample } & Curcumin & SDS & TritonX-100 & CTAB \\
\hline 1 & Collagen & $53 \cdot 1$ & $53 \cdot 1$ & $53 \cdot 1$ & $53 \cdot 1$ \\
2 & Collagen-additive $(1: 1)$ & $60 \cdot 4$ & $49 \cdot 9$ & $49 \cdot 2$ & $53 \cdot 5$ \\
3 & Collagen-additive $(1: 10)$ & $54 \cdot 5$ & $41 \cdot 3$ & $41 \cdot 9$ & $48 \cdot 6$ \\
4 & Collagen-additive $(1: 100)$ & $48 \cdot 5$ & $32 \cdot 2$ & $38 \cdot 2$ & $42 \cdot 2$ \\
\hline
\end{tabular}

collagen leads to increase followed by decrease of surface tension as presented in table 1. On the other hand, the addition of varying amounts of surface active agents to suspension of collagen leads to a gradual decrease in the static surface tension with increasing concentration of surface active agents as presented in table 1 . The effect of concentration of additives on the static surface tension of collagen can be understood in terms of adsorptive processes at local sites without only change in the hydrated structures at lower concentrations and with some changes in hydrated structures in higher concentrations. In other words, the effects of external additives on the molecular assemblies of collagen are some what complex. External agents like surfactants being ionic groups, could influence the charged dipoles of solvent (water) and collagen over long distance ranges. Curcumin on the other hand is expected to influence collagen in systems through covalent and non-covalent short range interactions.

Fibril formation of collagen is known to implicate supra-assembly and ordering of collagen molecules. Such an ordering is expected to lower their net surface charges. With varying concentrations of surfactants, adsorption of surfactants into collagen followed by aggregation of collagen-surfactant adducts is also expected. Lowering of surface tensions due to the action of surfactants on solvent is also a competitive phenomenon.

It is known that pure curcumin is not surface active and collagen is not strongly surface active. However, a synergistic effect of collagen and curcumin seems to lead to enhanced surface activity in the protein. It has been shown that curcumin has three $\mathrm{pKa}$ values at $8.38,9.88$ and 10.51 in aqueous solution, corresponding to deprotonation of the three hydroxyl groups (figure 1). ${ }^{18,19}$ Therefore, curcumin is fully protonated at $\mathrm{pH} 6$ to form the highly positively charged species. Therefore interaction of this species with the negative charges on the protein is highly likely. These attractive interactions allow curcumin to remain in close proximity to the protein and contribute greatly to its stabilization. Possibly a slight unfolding of the protein with less hydrophobic association makes the collagen more surface active.

The kinetics of fibril formation of collagen after crosslinking with curcumin was carried out and the results are shown in figure 6 . The rate of fibril formation of collagen in the absence and presence of curcumin is $7.01 \pm 0.02$ and $5.27 \pm 0.03$ (collagen:curcumin ratio $1: 1$ ), respectively. It can be seen that there is a decrease in the rate of fibril formation of collagen after crosslinking with curcumin. The protein-protein interaction is hindered due to the presence of curcumin. On increasing the concentration of collagen: curcumin to $1: 100$, the rate of fibril formation is enhanced. This could be due to the increased surface activity as seen from surface tension measurements. Earlier curcumin had been shown to enhance the thermal stability of collagen by $26^{\circ} \mathrm{C} .{ }^{17}$ The dimensional stability of collagen seems to be maintained on treatment with curcumin.

In the QCM measurements, injection of each sample of collagen (with and without the different agents) into the cell caused a frequency change, indicating adsorption of collagen onto the crystal. Data are from one representative experiment of at least three performed. It is assumed that Sauerbrey's equation holds good for the concentration of collagen used where the $-\Delta f$ is proportional to mass. Known volumes of the collagen and collagen with curcumin, SDS, CTAB and TritonX-100 were injected into the QCM cell for each experiment for measuring the frequency change as a function of time. Figure 7 shows the rate of change in $\Delta f$ for collagen adsorbing to the quartz crystal surface. Here for the sake of brevity, only the plot for pure collagen adsorbing onto the quartz surface is shown. All the frequency vs time plots were fitted to a sigmoidal function of the form, 


$$
y=A_{2}+\left(A_{1}-A_{2}\right) /\left(1+\exp \left(\left(x-x_{0}\right) / \mathrm{d} x\right)\right),
$$

where $A_{1}$ indicates the initial frequency value and $A_{2}$ denotes the final frequency value after adsorption reaches equilibrium, $x_{0}$ the point of inflexion in the plot and $\mathrm{dx}$ the time constant.

The initial frequency value varied between \pm 25 for both collagen and crosslinked collagen. It can be seen from table 2 that the final frequency value $\left(A_{2}\right)$ after adsorption is more for native protein when

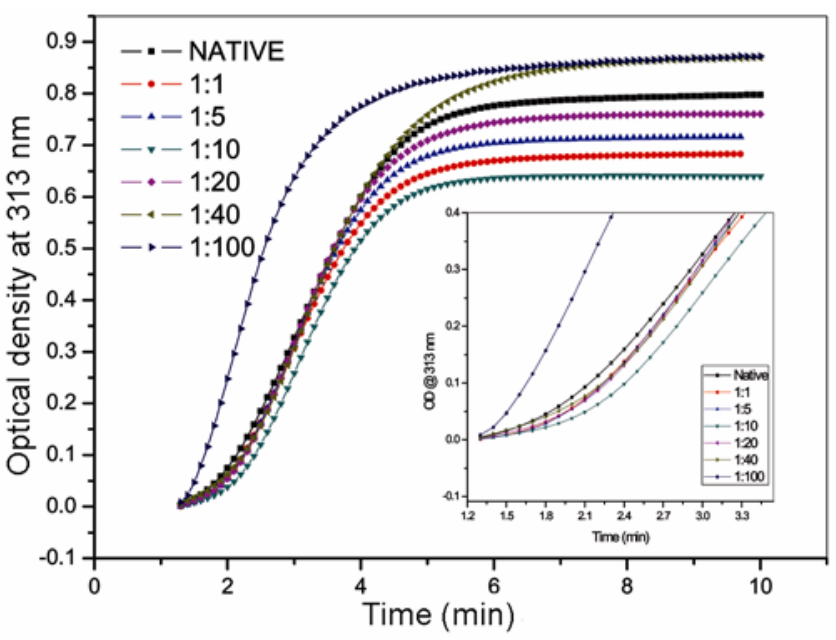

Figure 6. Turbidity measurement plot of RTT collagen and curcumin crosslinked RTT collagen @,313 nm, insert: showing the difference in lag phase due to addition of curcumin.

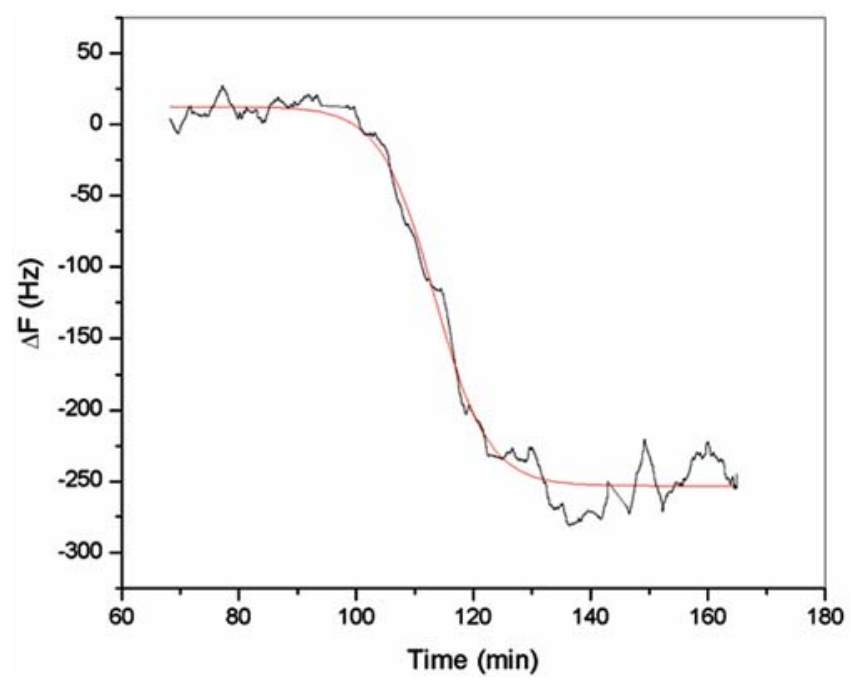

Figure 7. Frequency shift as a function of time for adsorption of RTT collagen on thiol coated substrate (black line indicates experimental data, red line indicates sigmoidal fit $),\left(T=25^{\circ} \mathrm{C}\right)$. compared to collagen +curcumin and collagen + SDS and less when compared to collagen + Triton X-100 and collagen + CTAB. Curcumin crosslinks with collagen through hydrogen bonds, which may lead to change in the water structure of the protein. Hence, there is more adsorption (frequency and mass being inversely proportional). In the case of SDS, there is charge compensation possible as it is an anionic surfactant, which could change the water structure of protein thus leading to more adsorption. Without considering the differences in the experimental systems, collagen and collagen + Triton X-100 showed nearly same rate of adsorption, while curcumin, SDS and CTAB showed faster rate of adsorption of protein to the surface. Triton X-100 being non ionic does not influence the rate of adsorption as other additives, which posses a charge.

It can be seen from the results that the dynamic surface tension remains almost constant for native collagen, whereas for the collagen treated with curcumin there is a decrease with time. In the cases of the surfactants with collagen for concentrations of CTAB, SDS and Triton X-100 below their CMCs, this synergistic effect is seen suggesting that the particular morphology and aggregation that may take place at air/solution interface may result from a balance between the burial of the hydrophobic groups, and the exposure and solvation of the more charges or polar groups at the surface. Results of QCM show that the larger the synergistic effect between collagen + additive, the larger is the frequency change as is seen from table 2. Thus, the tendency for collagen to express larger surface activity leads to better adsorption on the surface.

\section{Conclusions}

The focus of the work has been to understand the organization of collagen at interface. A not so surface active fibrous protein like collagen is shown to become surface active in the presence of additives. This can also lead to easy preparation of thin films of the protein. Since curcumin can bring about significant increase in thermal stability with minimal alteration in the dimensional stability of collagen it offers tremendous potential to be an effective stabilizing agent for collagen, which finds varied biomedical engineering applications. In conclusion, modulation of aggregation at interface and possible changes in reasonable folding processes in collagen due to these additives may be an approach that could 
Table 2. Initial, final and time constant value of frequency and surface tension value for collagen and collagen with additives on decane thiol coated substrate.

\begin{tabular}{lrrrrr}
\hline Property & Collagen & $\begin{array}{c}\text { Collagen }+ \\
\text { curcumin }\end{array}$ & $\begin{array}{c}\text { Collagen } \\
\text { SDS }\end{array}$ & $\begin{array}{c}\text { Collagen }+ \\
\text { Triton X-100 }\end{array}$ & $\begin{array}{c}\text { Collagen }+ \\
\text { CTAB }\end{array}$ \\
\hline Initial value (A1) & $12 \cdot 48$ & -0.49 & -0.96 & $27 \cdot 12$ & $-23 \cdot 44$ \\
Final value (A2) & $-253 \cdot 28$ & $-356 \cdot 43$ & $-306 \cdot 38$ & $-94 \cdot 67$ & $-132 \cdot 24$ \\
Rate $\left(x_{0}\right)$ & $113 \cdot 35$ & 51.22 & 53.58 & 113.53 & 91.98 \\
$\mathrm{~d} x$ & $4 \cdot 59931$ & $2 \cdot 46$ & $9 \cdot 65$ & $2 \cdot 02$ & $4 \cdot 17$ \\
$R^{2}$ & 0.98 & 0.99 & 0.99 & 0.92 & 0.90 \\
$\gamma$ & $53 \cdot 1$ & 48.5 & $32 \cdot 2$ & $38 \cdot 2$ & $42 \cdot 2$ \\
\hline
\end{tabular}

be explored for possible applications in bio-materials and for delivery of protein-drug complexes.

\section{References}

1. Sionkowska A 2000 Polym. Degrad. Stab. 68147

2. Gross J, Highberger J H, Schmitt F O 1952 Proc. Soc. Exp. Biol. Med. 80462

3. Jackson D S and Fessler J H 1955 Nature 17669

4. Nimni M E, Harkness R D 1988 in Collagen (ed.) M E Nimni (Boca Raton: CRC Press), vol. 1, pp. $1-4$

5. Trelstad R, Hayashi K and Gross J 1976 Proc. Nat. Acad. Sci. USA 734027

6. Goh M C, Paige M F, Gale M A, Yadegari I, Edirisinghe M and Strzelczyk J 1997 Physica A239 95

7. Köster S, Evans H M, Wong J Y and Pfohl T 2008 Biomacromolecules 9199

8. Uversky V N and Fink A L 2004 Biochim. Biophys. Acta 1698131
9. Dickinson D A, Levonen A L, Moellering D R, Arnold E K, Zhang H, Darley-Usmar V M and Forman H J 2004 Free Radic. Biol. Med. 371152

10. Ruby A J, Kuttan G and Babu K D 1995 Cancer Lett. 9479

11. Aggarwal B B, Kumar A and Bharti AC 2003 Anticancer Res. 23363

12. Jankun E S, McCabe N P, Selman S H and Jankun J 2000 Int. J. Mol. Med. 6521

13. Chandrakasan G, Torchia D A and Piez K A 1976 J. Biol. Chem. 2516062

14. Woessner Jr J F 1961 Arch. Biochem. Biophys. 93440

15. Sauerbrey G 1959 Z. Phys. 155206

16. Fathima N N and Dhathathreyan A 2009 Int. J. Biol. Macromol. 45274

17. Fathima N N, Devi S R, Rekha K B and Dhathathreyan A 2009 J. Chem. Sci. 121509

18. Bernabe-Pineda M, Ramirez-Silva M T, RomeroRomo M, Gonzalez-Vergara E and Rojas-Hernandez 2004 Spectrochim. Acta A60 1091

19. Tønnesen H H and Karlsen J Z 1985 Lebensm.Unters. Forsch. 180402 